\title{
Regulation of dendritic branching by Cde42 GAPs
}

\author{
Sergi Simó and Jonathan A. Cooper ${ }^{1}$ \\ Fred Hutchinson Cancer Research Center, Seattle, Washington 98109, USA
}

Nerve cells form elaborate, highly branched dendritic trees that are optimized for the receipt of synaptic signals. Recent work published in this issue of Genes \& Development by Rosario and colleagues (pp. 1743-1757) shows that a Cdc42-specific GTPase-activating protein (NOMA-GAP) regulates the branching of dendrites by neurons in the top layers of the mouse cortex. The results raise interesting questions regarding the specification of arbors in different cortical layers and the mechanisms of dendrite branching.

Early neuroanatomists distinguished many different types of neurons based solely on the morphology of the cell body and the patterning and targeting of their informationcarrying processes: axons and dendrites (Ramon y Cajal 1990). We now know that neurons also differ in their developmental origins, gene expression patterns, and electrophysiology, but morphology remains a useful basis for neuron identification. Dendrites are particularly distinctive. The branching patterns of dendrites, known as arbors because of their tree-like form, range from the nearly twodimensional vertical espaliers of cerebellar Purkinje cells to the freely branching bushes of basket cells and the horizontal disc arbors of retinal ganglion cells (Ramon y Cajal 1990). The arbor takes shape before neural networks are established, so it is presumably determined by autonomous developmental programs and exogenous nonsynaptic signals that together regulate the number, direction, and growth rate of primary dendrites and the positions and lengths of branches (Scott and Luo 2001; Urbanska et al. 2008). Later, new branches may sprout or old branches may be pruned in response to synaptic inputs on the "use it or lose it" principle. However, the regulation of the initial branching pattern remains poorly understood.

\section{Dendritogenesis in the neocortex}

The neocortex-the "thinking" part of the mammalian brain-is composed of layers of morphologically distinct neurons. More than three-quarters of cortical neurons are

[Keywords: Cdc42; NOMA-GAP; cofilin; dendritic branching; dendritic complexity; neocortical development; neuronal differentiation]

${ }^{1}$ Corresponding author

Email jcooper@fhcrc.org

Article is online at http://www.genesdev.org/cgi/doi/10.1101/gad.199034.112. projection neurons that extend long axons to other parts of the brain and deliver excitatory signals, while the remainder are interneurons, making local, inhibitory connections. The projection neurons are born below the cortex proper and migrate upward to the top of the cortex (Ayala et al. 2007). Successive neurons migrate beyond their predecessors, so early-born neurons move short distances and end up deep in the neocortex, below lateborn neurons at the top (Fig. 1A).

The genesis of axons and dendrites by cortical projection neurons is temporally and spatially regulated. Axonogenesis starts soon after a neuron is born and committed, during the time that the neuron itself is still migrating from its birthplace to the growing cortical plate. On the other hand, dendrites form after migration ends. Many projection neurons are "pyramids," with a prominent apical dendrite. This may derive from the leading process that explores the environment ahead of the neuron during its migratory phase (Hatanaka and Murakami 2002). When an early-born neuron reaches its layer and stops moving, the leading process must lengthen in order to maintain its connections with the top of the growing cortical plate (Fig. 1A, blue neuron in layer 5). Other primary dendrites extending from the cell body are presumably formed de novo after migration stops. Late-born neurons in layers 2 and 3 also have an apical dendrite that may derive from the leading process and basal dendrites that form de novo (Fig. 1A, red neuron).

\section{Dendrite branching}

We can imagine two ways that dendrites may branch (Fig. 1B). (1) The leading tip of a growing primary dendrite may fork, and both tines of the fork may extend simultaneously. (2) Side branches may form at some distance behind the growth tip. Distinguishing these possibilities requires sequential imaging of individual cells over time. Such studies have not been reported in the neocortex but have been done using hippocampal slices ex vivo and the optic tectum of live tadpoles and zebrafish (Dailey and Smith 1996; Wu et al. 1999; Niell et al. 2004; Urbanska et al. 2008). In these experiments, primary dendrites form first, and side branches emerge later. The side branches start out as transient slender projections, or filopodia, that emerge along the shaft of a primary dendrite at some 
A

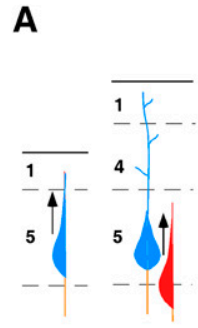

E14.5 E16.5

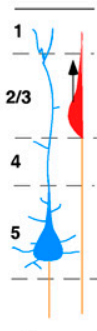

E18.5

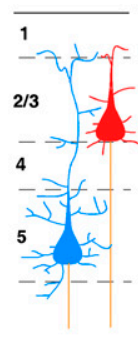

birth

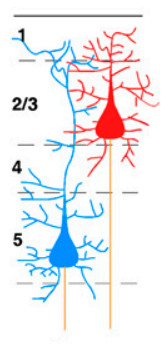

P5

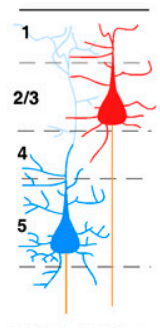

NOMA-GAP -/-
B
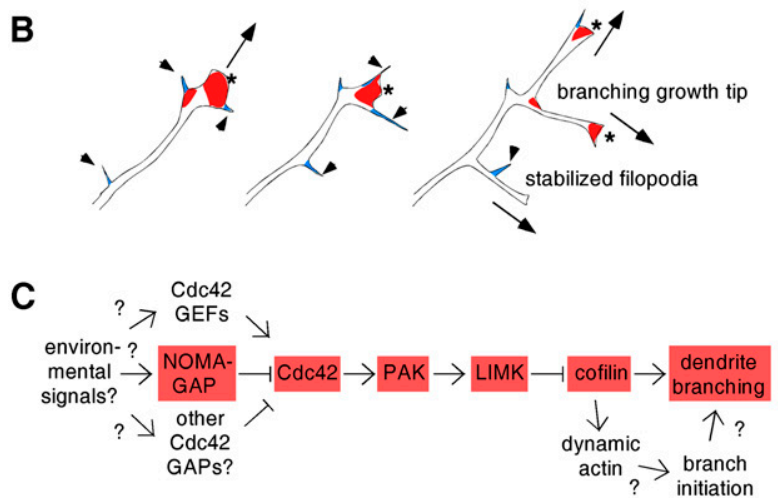

Figure 1. Dendrite development in the neocortex. $(A)$ Development of normal and NOMA-GAP mutant cortex. Time course of development with approximate embryo ages. (E14.5 [embryonic day 14.5]) Layer 5 cells (blue) arrive below layer 1, trailing an axon (orange) behind. (E16.5) The leading processes of layer 5 cells extend as the cortex grows, with layer 4 cells (not shown) inserting between layer 5 and layer 1 . Layer $2 / 3$ cells are entering from below. (E18.5) Layer $2 / 3$ cells (red) arrive between layer 4 and layer 1 . Primary dendrites have formed layer 5 cells. (Birth) Primary dendrites have formed layer $2 / 3$ cells. Branches forming on layer 5 cells. (P5 [postnatal day 5]) Branches forming on layer $2 / 3$ dendrites. Dendrites continue to remodel in response

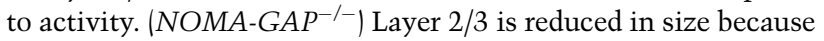
the cells have normal primary dendrites but few branches. Layer 5 cells have normal dendritic branching close to the cell body (i.e., in layer 5). The extent of branching of layer 5 cell dendrites in layer $2 / 3$ is not known (pale blue). (B) Possible mechanisms of dendrite branching by either branching of the growth tip or stabilization of filopodia on the dendrite shaft. $\left(^{*}\right)$ Lamellipodia; (arrowheads) filopodia; (arrows) direction of growth. (C) Signaling pathways that regulate dendrite branching in layer $2 / 3$. Steps established by Rosario et al. 2012 are highlighted in red.

distance behind the growth tip. Filopodia are highly dynamic actin structures and can retract or grow. A filopodium can become a dendrite branch if it is stabilized. Stabilization coincides with, and may be caused by, the formation of synapses on the incipient branch. The stabilization of side branches by postsynaptic signaling presumably occurs by a process similar to the stabilization of dendritic spines, most likely involving calcium signaling (Konur and Ghosh 2005). Side branch formation thus requires dynamic actin assembly and disassembly for the formation and remodeling of filopodia, followed by stabilization of the actin cytoskeleton as the branching pattern develops.

\section{RhoGTPases and the actin cytoskeleton}

RhoGTPases are guanine nucleotide-binding proteins that function as molecular switches by cycling between an active GTP-bound state and an inactive GDP-bound state (Etienne-Manneville and Hall 2002). The GTPbound state binds effector molecules that regulate actin assembly and disassembly processes.

The most intensively studied members of this large family are RhoA, Rac1, and Cdc42 (Etienne-Manneville and Hall 2002). They bind distinct effectors and are targeted to different subcellular membrane compartments by characteristic lipid modifications. Their activities are constantly regulated by at least three different sets of regulators; namely, GEFs (guanine nucleotide exchange factors), GAPs (GTPase-activating proteins), and GDIs (guanine nucleotide dissociation inhibitors). GEFs activate a RhoGTPase by stimulating the exchange of GTP for GDP, while GAPs enhance the intrinsic rate of GTP hydrolysis of the RhoGTPase, causing inactivation. GDIs interfere with GEF activity and sequester inactive RhoGTPases in specific areas of the cell. Notably, there are many more GAPs and GEFs than there are RhoGTPases, which makes for a bewildering variety of possible control points.

As a broad generalization, Rho family GTPases regulate the actin cytoskeleton. In many cell types, Cdc42 stimulates filopodia (Fig. 1B, arrowheads), Rac1 stimulates lamellipodia (Fig. 1B, asterisks), and RhoA stimulates contractile stress fibers (Hall 1998). Accordingly, RhoA, Rac1, and Cdc42 play key roles in the growth and branching of axons and dendrites (Govek et al. 2005; Urbanska et al. 2008). The first evidence came from studies using neuronal cell lines (Govek et al. 2005). Commonly used neuronal lines can undergo the initial steps of neuronal differentiation and extend neurites, which are the uncommitted precursors of dendrites and axons. In these in vitro systems, Cdc42 and Racl induce neurite growth and branching, while Rho inhibits (Govek et al. 2005). Further work in several in vivo systems has confirmed that Racl stimulates and Rho inhibits dendrite growth and branching, but the role of Cdc42 is less clear (Ruchhoeft et al. 1999; $\mathrm{Ng}$ et al. 2002). Expression of activated mutant Cdc42 inhibits dendrite growth in the Drosophila peripheral nervous system (Luo et al. 1994) but induces dendrites in Xenopus optic tectal neurons (Li et al. 2000). Loss of Cdc42 increased the length but did not alter the complexity of dendrites of specific neurons in the Drosophila CNS (Scott et al. 2003). These apparently contradictory results suggest that the role of Cdc42 is more complex and may vary in different types of neurons. In addition, the function of Cdc42, and possibly other Rho GTPases, may not depend on the permanent activation or inhibition state, but on the rate and localization of cycling between the GDP- and GTP-bound states. As a consequence, GEFs, GAPs, and GDIs acquire key roles in deciding where within the cell, when, and for how long Cdc42 is active or inactive.

\section{NOMA-GAP regulates dendritic branching}

In this issue of Genes \& Development, Rosario et al. (2012) identified NOMA-GAP as a Cdc42-specific GAP 
that is required for proper dendritic development in the upper layers of the neocortex. They found that homozygous deletion of the NOMA-GAP gene has little or no effect on neurogenesis, neuron fate specification, cell migration, axonogenesis, or the number of primary dendrites. However, when NOMA-GAP is absent, there is a marked reduction in the number of dendrite branches of layer $2 / 3$ neurons, resulting in a simpler dendritic tree. Cdc42 activity is increased in the mutant cortex. In an elegant and convincing series of experiments, Rosario et al. (2012) go on to show that the phenotype of a NOMA-GAP mutant is rescued by heterozygous deletion of $C d c 42$, thus showing genetically that the role of NOMA-GAP is to inhibit Cdc42. They show that downstream from Cdc42, a $\mathrm{Cdc} 42$ effector, the protein kinase PAK1, is activated when NOMA-GAP is absent and that a PAK1 substrate, another protein kinase, LIMK, is also activated. LIMK is known to phosphorylate and inhibit an actin severing/ depolymerizing molecule, cofilin (Arber et al. 1998). Deletion of NOMA-GAP caused a significant increase in phosphorylated cofilin, particularly in the upper layers. In a final satisfying experiment, the investigators expressed a nonphosphorylatable mutant of cofilin in the NOMAGAP mutant cortex and again rescued the phenotype. In sum, the study provides thorough documentation for a signaling pathway linking NOMA-GAP through Cdc42, PAK1, LIMK, and cofilin to dendrite branching (Fig. 1C).

\section{Layer-specific dendritic complexity}

One interesting aspect of the NOMA-GAP mutant phenotype is that the reduction in dendritic complexity is apparent in layer $2 / 3$ neurons but not in layer 5 neurons, at least within $300 \mu \mathrm{m}$ of the cell body, which was as far as the investigators could measure (Rosario et al. 2012). This raises the question of how NOMA-GAP regulates dendrite branching in layer $2 / 3$ and not layer 5 , given that NOMA-GAP is expressed in all layers. Here we consider that NOMA-GAP may be required more in one layer than another because (1) other Cdc42-GAPs are expressed in some layers and can substitute for NOMA-GAP or (2) Cdc42 is activated more in some layers than others as a result of, for example, layer-specific environmental signals or differentially expressed Cdc42-GEFs.

\section{Potential redundancy: overlap between NOMA-GAP and RICS}

An obvious potential source of redundancy for NOMAGAP is its close relative, RICS. Both proteins have been studied extensively under various names (NOMA-GAP/ ARHGAP33/SNX26/TCGAP and RICS/PX-RICS/ARH GAP32/Grit/p200RhoGAP/GC-GAP). They are expressed in the same brain regions during development and are highly similar in structure and protein-protein interactions (Nakamura et al. 2002; Chiang et al. 2003; Hayashi et al. 2007; Rosario et al. 2007). Both NOMA-GAP and Phox homology (PX)-RICS (the main isoform in developing brains) contain an $\mathrm{N}$-terminal phosphoinositide-binding PX domain, through which they localize to cell membranes.
After the PX domain, there is a SH3 domain, which typically binds to proline-rich peptides, followed by the RhoGAP domain, and finally, several proline-rich sequences localized in the C-terminal part of the protein. Many of the C-terminal proline sequences fit the consensus for binding to $\mathrm{SH} 3$ domains, suggesting that NOMA-GAP and RICS might autoregulate. Indeed, fulllength NOMA-GAP and RICS are less active than their isolated GAP domains when overexpressed (Nakamura et al. 2002; Chiang et al. 2003). The proline sequences also bind to a large number of SH3 domain-containing signaling proteins. The neurotrophin receptor TrkA also binds to the $\mathrm{C}$ termini of both NOMA-GAP and RICS. These similarities suggest that NOMA-GAP and RICS may well have overlapping functions.

Genes with overlapping functions are typically redundant with each other. A RICS knockout mouse was made, but no phenotype was detected (Nasu-Nishimura et al. 2006). However, RICS knockout neurons had more active Cdc42 and developed longer neurites when cultured in vitro, suggesting a role for RICS in dendritogenesis (NasuNishimura et al. 2006). Very recently, RNAi was used to knock down RICS in developing rat cortex (Long et al. 2012). Intriguingly, RICS knockdown reduced the dendritic complexity of layer 2/3 neurons (Long et al. 2012), similar to the phenotype of NOMA-GAP knockout mice. Thus, it is possible that closer examination of RICS knockout mice might reveal defects in dendrite branching that were previously overlooked.

If RICS has functions similar to NOMA-GAP and is expressed in the same cells, why do the knockouts and knockdowns have any phenotypes at all? One possible explanation is that, despite their many similarities, the NOMA-GAP and RICS proteins may have different downstream effectors and upstream regulators. In this regard, differences in GAP activity and binding partners of NOMA-GAP and RICS have been reported, but unfortunately, the two proteins were not compared side by side by the same experimenters. For example, NOMA-GAP was reported to stimulate hydrolysis of Cdc42.GTP and Rac1.GTP faster than RhoA.GTP (Chiang et al. 2003), yet it binds more tightly to Cdc42.GTP than to Rac1.GTP or RhoA.GTP (Rosario et al. 2007). Different researchers reported that RICS stimulates GTP hydrolysis equally by Cdc42, Rac1, or RhoA (Moon et al. 2003) or that RICS prefers Cdc42 over Racl or RhoA as a substrate (Nakamura et al. 2002). In vivo, both NOMA-GAP and RICS are linked predominantly to Cdc42 (Chiang et al. 2003; Okabe et al. 2003; Hayashi et al. 2007; Rosario et al. 2012). RICS binds to $\beta$-catenin, E-Cadherin, N-Cadherin, PSD-95/DLG4, and a variety of SH3 domain proteins (Okabe et al. 2003). However, these interactions may not be unique, since binding to NOMA-GAP has not been tested.

Both NOMA-GAP and RICS are under upstream control by tyrosine phosphorylation. NOMA-GAP can be phosphorylated in at least two regions; namely, the GAP domain by the Src-family kinase Fyn, and the C-terminal tail by TrkA (Liu et al. 2006; Rosario et al. 2007). In NGFstimulated PC12 cells, Fyn-dependent phosphorylation of NOMA-GAP inhibited the GAP activity and promoted 
neurite outgrowth in a Cdc42-dependent manner (Liu et al. 2006). NGF also stimulated TrkA-dependent phosphorylation of the C-terminal tail of NOMA-GAP. This recruited Shp2, activated Erk5, and stimulated neurite outgrowth (Rosario et al. 2007). Similarly, RICS can be phosphorylated by TrkA and Src kinases (Nakamura et al. 2002; Moon et al. 2003), although the functional significance for dendrite outgrowth or branching is less clear. RICS can also be phosphorylated by CaMKII and possibly by EGFR, although it is not clear whether the EGFRdependent phosphorylation is direct or indirect through Src activation (Nakamura et al. 2002; Okabe et al. 2003).

Given this complexity, an important task for the future will be to systematically compare NOMA-GAP and RICS in the same binding and biochemical assays and to test the effects of single and double knockdown or knockout on dendrite branching in vivo.

\section{Transcriptional regulation of the Cdc42 pathway}

Differential expression of other Cdc42 GAPs or GDIs could suppress the requirement for NOMA-GAP in deeplayer neurons. In this regard, there are, of course, many differences in gene expression between neurons in different layers (Ayoub et al. 2011), and any of the large number of Cdc42 GAPs or GDIs could compensate when NOMAGAP is absent from layer 5 neurons. However, recent results suggest candidate transcription factors that may regulate the layer-specific expression of Rho regulators.

In flies, the transcription factor Cut controls the dendrite morphology of post-mitotic neurons (Grueber et al. 2003). Interestingly, in mice, Cux1 and Cux2, which are the vertebrate homologs of Cut, are specifically expressed in the top layers of the cortical plate. Moreover, analysis of single or double Cux1/Cux2 mutant mouse brains showed reduced dendritic complexity just in the top layers of the cortical plate, similar to the NOMA-GAP mutant (Cubelos et al. 2010; Rosario et al. 2012). These data suggest a model in which Cux1 and Cux2 might regulate expression of Cdc42 regulators, giving a possible explanation for the layer-specific phenotype in NOMA-GAP mutant mice.

Another possible mechanism to generate diversity is through the specific expression of microRNAs (miRs). miR-132 is enriched in the brain and targets RICS in cultured hippocampal neurons, stimulating dendrite extension and branching (Vo et al. 2005). In silico, at least three different microRNAs may target NOMA-GAP (miR-544, miR-31, and miR-490). Analysis of their capacity to recognize and target NOMA-GAP mRNA as well as their layer distribution during late development might help to understand the highly restrictive phenotype in NOMA-GAP mice.

\section{Input signals: layer-specific activation of Cdc42 in the developing cortex}

It is self-evident that Cdc42 inactivation by GAPs is only necessary if Cdc42 is activated. Therefore, the increased requirement for NOMA-GAP in upper cortical layers could be a reflection of increased activation of Cdc42
GEFs in these neurons. Indeed, arborization is known to be regulated by diffusible cues, cell-cell contacts, and neuronal activity, which vary layer by layer (Urbanska et al. 2008). Three diffusible signals-Reelin, Sema3A, and neurotrophins-are of particular interest because their expression is layer-specific and they regulate tyrosine kinases that modify NOMA-GAP and RICS and also regulate GEFs for Cdc42.

Reelin is expressed primarily in layer 1 of the developing brain but diffuses down through the cortical plate (D'Arcangelo et al. 1995; Jossin et al. 2007). In vitro, Reelin stimulates dendrite growth, and dendritic complexity is reduced in vivo in Reelin heterozygous mutant mice (Niu et al. 2004). Reelin signals by activating Src family tyrosine kinases Src and Fyn, which are known regulators of NOMA-GAP and RICS (Arnaud et al. 2003; Moon et al. 2003; Liu et al. 2006; Rosario et al. 2007). Tyrosine kinases stimulate many Rho family GEFs by phosphorylation or membrane recruitment (Schiller 2006). The increased Reelin concentration near the top of the cortical plate could therefore stimulate Cdc42 GTP loading in layer $2 / 3$ neurons more than in layer 5, necessitating increased NOMA-GAP activity to compensate.

Sema3A was identified as a chemoattractant for dendrites using assays in which dissociated cortical neurons were plated onto live cortical slices (Polleux et al. 2000). Sema3A is produced near the top of the cortical plate and forms a gradient from top to bottom. The gradient stimulates the growth of apical dendrites toward the source of Sema3A. Sema3A also stimulates dendritic branching, in part through Fyn (Morita et al. 2006). Curiously, Sema3A induces dendrite collapse in cultured neurons, seemingly the opposite of its main role in vivo (Sasaki et al. 2002). These results emphasize the importance of in vivo experiments to control for the artificial environment imposed by in vitro culture.

The neurotrophins BDNF, NT-3, and NT-4 have complicated combinatorial effects on dendrite growth and arborization. BDNF stimulates dendrite growth by layer 4 neurons and inhibits layer 6 neurons (McAllister et al. 1995, 1997). Reciprocally, NT-3 inhibits layer 4 neurons and stimulates layer 6 neurons. NT-4 stimulates layer 6 dendrites early in development and layer $2 / 3$ neurons later (McAllister et al. 1995; Wirth et al. 2003). As discussed above, neurotrophin receptors (Trks) phosphorylate RICS and NOMA-GAP (Nakamura et al. 2002; Rosario et al. 2007) and also activate a variety of Rho family GEFs (Schiller 2006), so the effects of neurotrophins on dendritogenesis could be mediated by Cdc42 GEFs and GAPs. Interestingly, one mechanism for activation of Racl, and potentially Cdc42, is through the WAVE complex (Pollard et al. 2000). One component of the WAVE complex, Nap1, is induced by BDNF in culture and is expressed in vivo when neurons stop migrating and start differentiation (Yokota et al. 2007). Like NOMA-GAP, Nap1 is needed for dendritic arborization. Taken together, these results suggest a variety of ways in which neurotrophins could interact with Cdc42 in determining the layer specificity of dendritic arborization. 


\section{Cdc42, actin dynamics, and dendrite branching}

In principle, Cdc42 could impact dendrite branching through a number of different mechanisms. One is by regulating the polarity of growing dendrite processes. Cdc42 focuses actin polymerization to a restricted region of the cell membrane, thus regulating the polarity of cell migration and the site of axon emergence (EtienneManneville and Hall 2002). These processes are conceptually similar to maintaining a single point of growth at the tip of a dendrite, so high Cdc42 activity would inhibit branching, as observed in the NOMA-GAP mutant. However, these Cdc42 functions involve atypical protein kinase $\mathrm{C}$ and the Par complex, as opposed to the PAK1, LIMK, cofilin pathway used by NOMA-GAP (Rosario et al. 2012). Therefore, we need to understand how cofilin regulates branching.

Cofilin is an actin-severing protein. It interacts with actin filaments, breaks them, and associates with free ends (Pollard et al. 2000). In this way, it dismantles actin filaments. However, it also regenerates a pool of actin subunits for recycling, so cofilin actually promotes actin polymerization. The net effect is to stimulate actin dynamics. Thus, inhibition of cofilin by Cdc42PAK-LIMK stabilizes existing filaments and inhibits the growth of new ones (Arber et al. 1998), and cofilin knockout interferes with neurite formation in vivo and in vitro (Bellenchi et al. 2007). In addition, Cdc42 binds to and activates N-WASP, which stimulates actin filament assembly, and diaphanous-related formin 3 , which promotes the growth of parallel actin bundles (Pollard et al. 2000; Peng et al. 2003). Together, these Cdc42 effectors generate filopodia. The implication is that the activation of Cdc42 by NOMA-GAP deletion would induce filopodia via N-WASP and formins but, at the same time, reduce actin dynamics by inhibiting cofilin. The ability of nonphosphorylatable mutant cofilin to rescue the NOMAGAP mutant implies that vigorous actin dynamics are critical for branching.

Clearly, understanding the exact mechanism by which NOMA-GAP, Cdc42, and cofilin regulate dendrite branching in vivo will require the development of techniques for following the differentiation of neocortical neurons in vivo or in slice culture over long periods of time. However, if we assume that the dendritic branches of cortical neurons arise from filopodia, as discussed above (Dailey and Smith 1996; Wu et al. 1999; Niell et al. 2004; Urbanska et al. 2008), then it seems probable that NOMA-GAP stimulates the initiation of dynamic filopodia. The conversion of transient filopodia into stable branches, however, would require inhibition of NOMA-GAP in order to slow down actin turnover. These considerations indicate that dynamic spatial and temporal regulation of Cdc42 GAPs and GEFs lies at the heart of dendrite arbor formation.

\section{Acknowledgments}

We thank Jihong Bai and Yves Jossin for their comments on the manuscript. We are supported by R01 NS080194 from the National Institute of Neurological Diseases and Stroke.

\section{References}

Arber S, Barbayannis FA, Hanser H, Schneider C, Stanyon CA, Bernard O, Caroni P. 1998. Regulation of actin dynamics through phosphorylation of cofilin by LIM-kinase. Nature 393: 805-809.

Arnaud L, Ballif BA, Forster E, Cooper JA. 2003. Fyn tyrosine kinase is a critical regulator of Disabled-1 during brain development. Curr Biol 13: 9-17.

Ayala R, Shu T, Tsai LH. 2007. Trekking across the brain: The journey of neuronal migration. Cell 128: 29-43.

Ayoub AE, Oh S, Xie Y, Leng J, Cotney J, Dominguez MH, Noonan JP, Rakic P. 2011. Transcriptional programs in transient embryonic zones of the cerebral cortex defined by high-resolution mRNA sequencing. Proc Natl Acad Sci 108: 14950-14955.

Bellenchi GC, Gurniak CB, Perlas E, Middei S, AmmassariTeule M, Witke W. 2007. N-cofilin is associated with neuronal migration disorders and cell cycle control in the cerebral cortex. Genes Dev 21: 2347-2357.

Chiang SH, Hwang J, Legendre M, Zhang M, Kimura A, Saltiel AR. 2003. TCGAP, a multidomain Rho GTPase-activating protein involved in insulin-stimulated glucose transport. EMBO I 22: 2679-2691.

Cubelos B, Sebastian-Serrano A, Beccari L, Calcagnotto ME, Cisneros E, Kim S, Dopazo A, Alvarez-Dolado M, Redondo JM, Bovolenta P, et al. 2010. Cux1 and Cux2 regulate dendritic branching, spine morphology, and synapses of the upper layer neurons of the cortex. Neuron 66: 523-535.

Dailey ME, Smith SJ. 1996. The dynamics of dendritic structure in developing hippocampal slices. J Neurosci 16: 2983-2994.

D'Arcangelo G, Miao GG, Chen SC, Soares HD, Morgan JI, Curran T. 1995. A protein related to extracellular matrix proteins deleted in the mouse mutant reeler. Nature 374: 719-723.

Etienne-Manneville S, Hall A. 2002. Rho GTPases in cell biology. Nature 420: 629-635.

Govek EE, Newey SE, Van Aelst L. 2005. The role of the Rho GTPases in neuronal development. Genes Dev 19: 1-49.

Grueber WB, Jan LY, Jan YN. 2003. Different levels of the homeodomain protein cut regulate distinct dendrite branching patterns of Drosophila multidendritic neurons. Cell 112: 805-818.

Hall A. 1998. Rho GTPases and the actin cytoskeleton. Science 279: 509-514.

Hatanaka Y, Murakami F. 2002. In vitro analysis of the origin, migratory behavior, and maturation of cortical pyramidal cells. J Comp Neurol 454: 1-14.

Hayashi T, Okabe T, Nasu-Nishimura Y, Sakaue F, Ohwada S, Matsuura K, Akiyama T, Nakamura T. 2007. PX-RICS, a novel splicing variant of RICS, is a main isoform expressed during neural development. Genes Cells 12: 929-939.

Jossin Y, Gui L, Goffinet AM. 2007. Processing of Reelin by embryonic neurons is important for function in tissue but not in dissociated cultured neurons. J Neurosci 27: 4243-4252.

Konur S, Ghosh A. 2005. Calcium signaling and the control of dendritic development. Neuron 46: 401-405.

Li Z, Van Aelst L, Cline HT. 2000. Rho GTPases regulate distinct aspects of dendritic arbor growth in Xenopus central neurons in vivo. Nat Neurosci 3: 217-225.

Liu H, Nakazawa T, Tezuka T, Yamamoto T. 2006. Physical and functional interaction of Fyn tyrosine kinase with a brainenriched Rho GTPase-activating protein TCGAP. I Biol Chem 281: 23611-23619.

Long H, Zhu X, Yang P, Gao Q, Chen Y, Ma L. 2012. Myo9b and RICS modulate dendritic morphology of cortical neurons. Cereb Cortex. doi: 10.1093/cercor/bhr378. 
Luo L, Liao YJ, Jan LY, Jan YN. 1994. Distinct morphogenetic functions of similar small GTPases: Drosophila Dracl is involved in axonal outgrowth and myoblast fusion. Genes Dev 8: 1787-1802.

McAllister AK, Lo DC, Katz LC. 1995. Neurotrophins regulate dendritic growth in developing visual cortex. Neuron 15: 791-803.

McAllister AK, Katz LC, Lo DC. 1997. Opposing roles for endogenous BDNF and NT-3 in regulating cortical dendritic growth. Neuron 18: 767-778.

Moon SY, Zang H, Zheng Y. 2003. Characterization of a brainspecific Rho GTPase-activating protein, p200RhoGAP. J Biol Chem 278: 4151-4159.

Morita A, Yamashita N, Sasaki Y, Uchida Y, Nakajima O, Nakamura F, Yagi $T$, Taniguchi $M$, Usui $H$, Katoh-Semba $\mathrm{R}$, et al. 2006. Regulation of dendritic branching and spine maturation by semaphorin3A-Fyn signaling. I Neurosci 26: 2971-2980.

Nakamura T, Komiya M, Sone K, Hirose E, Gotoh N, Morii H, Ohta Y, Mori N. 2002. Grit, a GTPase-activating protein for the Rho family, regulates neurite extension through association with the TrkA receptor and N-Shc and CrkL/Crk adapter molecules. Mol Cell Biol 22: 8721-8734.

Nasu-Nishimura Y, Hayashi T, Ohishi T, Okabe T, Ohwada S, Hasegawa Y, Senda T, Toyoshima C, Nakamura T, Akiyama T. 2006. Role of the Rho GTPase-activating protein RICS in neurite outgrowth. Genes Cells 11: 607-614.

Ng J, Nardine T, Harms M, Tzu J, Goldstein A, Sun Y, Dietzl G, Dickson BJ, Luo L. 2002. Rac GTPases control axon growth, guidance and branching. Nature 416: 442-447.

Niell CM, Meyer MP, Smith SJ. 2004. In vivo imaging of synapse formation on a growing dendritic arbor. Nat Neurosci 7: 254 260.

Niu S, Renfro A, Quattrocchi CC, Sheldon M, D'Arcangelo G. 2004. Reelin promotes hippocampal dendrite development through the VLDLR/ApoER2-Dab1 pathway. Neuron 41: 7184.

Okabe T, Nakamura T, Nishimura YN, Kohu K, Ohwada S, Morishita Y, Akiyama T. 2003. RICS, a novel GTPaseactivating protein for $\mathrm{Cdc} 42$ and Racl, is involved in the $\beta$-catenin-N-cadherin and $\mathrm{N}$-methyl-D-aspartate receptor signaling. J Biol Chem 278: 9920-9927.

Peng J, Wallar BJ, Flanders A, Swiatek PJ, Alberts AS. 2003. Disruption of the Diaphanous-related formin Drf1 gene encoding mDial reveals a role for Drf3 as an effector for Cdc42. Curr Biol 13: 534-545.

Pollard TD, Blanchoin L, Mullins RD. 2000. Molecular mechanisms controlling actin filament dynamics in nonmuscle cells. Annu Rev Biophys Biomol Struct 29: 545-576.

Polleux F, Morrow T, Ghosh A. 2000. Semaphorin 3A is a chemoattractant for cortical apical dendrites. Nature 404: $567-573$

Ramon y Cajal S. 1990. New ideas on the structure of the nervous system in man and vertebrates. MIT Press, Cambridge, MA.

Rosario M, Franke R, Bednarski C, Birchmeier W. 2007. The neurite outgrowth multiadaptor RhoGAP, NOMA-GAP, regulates neurite extension through SHP2 and Cdc42. J Cell Biol 178: $503-516$

Rosario M, Schuster S, Juttner R, Parthasarathy S, Tarabykin V, Birchmeier W. 2012. Neocortical dendritic complexity is controlled during development by NOMA-GAP-dependent inhibition of Cdc42 and activation of cofilin. Genes Dev (this issue). doi: $10.1101 / \mathrm{gad} .191593 .112$.

Ruchhoeft ML, Ohnuma S, McNeill L, Holt CE, Harris WA. 1999. The neuronal architecture of Xenopus retinal ganglion cells is sculpted by rho-family GTPases in vivo. J Neurosci 19: 8454-8463.

Sasaki Y, Cheng C, Uchida Y, Nakajima O, Ohshima T, Yagi T, Taniguchi M, Nakayama T, Kishida R, Kudo Y, et al. 2002. Fyn and Cdk5 mediate semaphorin-3A signaling, which is involved in regulation of dendrite orientation in cerebral cortex. Neuron 35: 907-920.

Schiller MR. 2006. Coupling receptor tyrosine kinases to Rho GTPases-GEFs what's the link. Cell Signal 18: 1834-1843.

Scott EK, Luo L. 2001. How do dendrites take their shape? Nat Neurosci 4: 359-365.

Scott EK, Reuter JE, Luo L. 2003. Small GTPase Cdc42 is required for multiple aspects of dendritic morphogenesis. J Neurosci 23: 3118-3123.

Urbanska M, Blazejczyk M, Jaworski J. 2008. Molecular basis of dendritic arborization. Acta Neurobiol Exp (Wars) 68: 264288.

Vo N, Klein ME, Varlamova O, Keller DM, Yamamoto $T$, Goodman RH, Impey S. 2005. A cAMP-response element binding protein-induced microRNA regulates neuronal morphogenesis. Proc Natl Acad Sci 102: 16426-16431.

Wirth MJ, Brun A, Grabert J, Patz S, Wahle P. 2003. Accelerated dendritic development of rat cortical pyramidal cells and interneurons after biolistic transfection with BDNF and NT4/5. Development 130: 5827-5838.

Wu GY, Zou DI, Rajan I, Cline H. 1999. Dendritic dynamics in vivo change during neuronal maturation. I Neurosci 19: 4472-4483.

Yokota Y, Ring C, Cheung R, Pevny L, Anton ES. 2007. Nap1regulated neuronal cytoskeletal dynamics is essential for the final differentiation of neurons in cerebral cortex. Neuron 54: 429-445. 


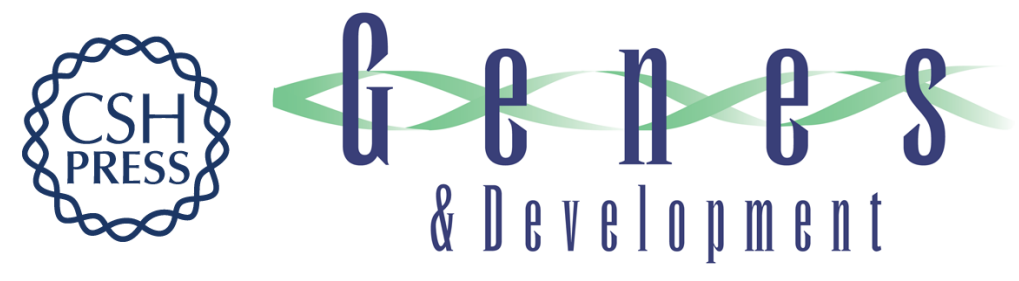

\section{Regulation of dendritic branching by Cdc42 GAPs}

\section{Sergi Simó and Jonathan A. Cooper}

Genes Dev. 2012, 26:

Access the most recent version at doi:10.1101/gad.199034.112

\section{Related Content Neocortical dendritic complexity is controlled during development by NOMA-GAP-dependent inhibition of Cdc42 and activation of cofilin Marta Rosário, Steffen Schuster, René Jüttner, et al. Genes Dev. August , 2012 26: 1743-1757}

References This article cites 44 articles, 18 of which can be accessed free at: http://genesdev.cshlp.org/content/26/15/1653.full.html\#ref-list-1

Articles cited in:

http://genesdev.cshlp.org/content/26/15/1653.full.html\#related-urls

\section{License}

Email Alerting

Service

Receive free email alerts when new articles cite this article - sign up in the box at the top right corner of the article or click here.

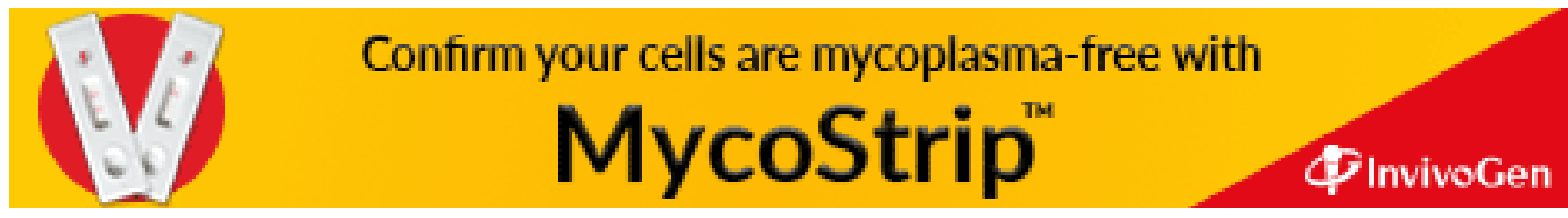

